\title{
SOME INEQUALITIES FOR A HYPERGEOMETRIC INTEGRAL
}

\author{
by T. M. FLETT \\ (Received 26th May 1971)
}

1. In various problems in harmonic analysis $(\mathbf{4}, 5,6)$, I have required an estimate for the integral for the hypergeometric function

$$
\int_{0}^{1}(1-t x)^{-a}(1-t)^{c-b-1} t^{b-1} d t=\frac{\Gamma(b) \Gamma(c-b)}{\Gamma(c)} F(a, b ; c ; x)
$$

in the case where $a>c-b>0, b>0$, and $0 \leqq x<1$ (the integral is then unbounded as $x \rightarrow 1-$ ). Although there are innumerable identities for hypergeometric functions, few inequalities for these functions seem to be known, $\uparrow$ and in estimating the integral (1) I employed ad hoc arguments that made no use of the theory of hypergeometric functions. The estimates obtained were adequate for my purposes, but were far from sharp, and the object of this note is to show that, by assembling a few known facts concerning hypergeometric functions, we can obtain sharp inequalities for the integral (1). We also give (in $\S 4$ ) several related inequalities that can be obtained by transforming the integral.

2. The properties of the hypergeometric function $F(a, b ; c ; x)$ that we require are contained in the following lemma.

Lemma. Let $a>c-b>0, b>0,0 \leqq x<1$, and let $G=G_{a, b, c}$ be the function with domain $[0,1]$ given by

$$
\begin{aligned}
G(x) & =(1-x)^{a+b-c} F(a, b ; c ; x) \quad(0 \leqq x<1), \\
G(1) & =\frac{\Gamma(c) \Gamma(a+b-c)}{\Gamma(a) \Gamma(b)}
\end{aligned}
$$

(so that $G(0)=1$ ). Then

(i) $G$ is continuous,

(ii) $G$ is strictly increasing if $a<c$, strictly decreasing if $a>c$, and constant if $a=c$,

(iii) $G$ is strictly convex if $a<c$ or $a>c+1$, is strictly concave if $c<a<c+1$, and is a polynomial of the first degree if $a=c+1$.

$\dagger$ The only study of inequalities for hypergeometric functions known to me is that of Erber (2). 
The function $G$ is obviously continuous on $[0,1[$, and it is familiar that $G(x) \rightarrow G(1)$ as $x \rightarrow 1-(3, \S 2.9(1),(2)$ and $\S 2.8(46))$. Further,

and

$$
G^{\prime}(x)=(c-a)(c-b) c^{-1}(1-x)^{a+b-c-1} F(a, b ; c+1 ; x),
$$

$G^{\prime \prime}(x)=(c-a)(c-a+1)(c-b)(c-b+1) c^{-1}(c+1)^{-1}$

$$
(1-x)^{a+b-c-2} F(a, b ; c+2 ; x)
$$

$(3, \S 2.8(24))$. Since $F(a, b ; c+k ; x)>0$ for $k=1,2$, and $c>b>0$, these two identities imply (ii) and (iii) respectively.

3. To simplify our notation in dealing with the integral (1), we now replace $c-b$ by a single symbol. We therefore write

$$
I(\alpha, \beta, \gamma, x)=\int_{0}^{1}(1-t x)^{-\alpha}(1-t)^{\beta-1} t^{\gamma-1} d t,
$$

where $\alpha>\beta>0, \gamma>0$, and $0 \leqq x<1$. Then

$$
I(\alpha, \beta, \gamma, x)=B(\beta, \gamma)(1-x)^{\beta-\alpha} G_{\alpha, \gamma, \beta+\gamma}(x),
$$

where $B$ is the Beta function. Since

$$
B(\beta, \gamma) G_{\alpha, \gamma, \beta+\gamma}(1)=B(\alpha-\beta, \beta),
$$

and since the Beta function is symmetric and decreasing in each variable, the results of the lemma yield the following results for $I(\alpha, \beta, \gamma, x)$.

Theorem. Let $\alpha>\beta>0, \gamma>0,0 \leqq x<1$. Then

(i) $I(\alpha, \beta, \gamma, 0)=B(\beta, \gamma)$, and $I(\alpha, \beta, \gamma, x) \sim B(\alpha-\beta, \beta)(1-x)^{\beta-\alpha}$ as $x \rightarrow 1-$,

(ii) $B(\lambda, \beta)(1-x)^{\beta-\alpha} \leqq I(\alpha, \beta, \gamma, x) \leqq B(\mu, \beta)(1-x)^{\beta-\alpha}$,

where $\lambda=\max \{\alpha-\beta, \gamma\}, \mu=\min \{\alpha-\beta, \gamma\}$. In particular,

(iii) if $\alpha=\beta+\gamma$, then $I(\alpha, \beta, \gamma, x)=B(\beta, \gamma)(1-x)^{-\gamma}$.

\section{Further}

(iv) if $\alpha=\beta+\gamma+1$, then

$$
\begin{aligned}
I(\alpha, \beta, \gamma, x) & =(1-x)^{\beta-\alpha}\{(1-x) B(\beta, \gamma)+x B(\alpha-\beta, \beta)\} \\
& =B(\beta, \gamma)(1-\beta x /(\beta+\gamma))(1-x)^{-\gamma-1},
\end{aligned}
$$

(v) if either $\alpha<\beta+\gamma$ or $\alpha>\beta+\gamma+1$, then for $0<x<1$

$$
I(\alpha, \beta, \gamma, x)<(1-x)^{\beta-\alpha}\{(1-x) B(\beta, \gamma)+x B(\alpha-\beta, \beta)\},
$$

while if $\beta+\gamma<\alpha<\beta+\gamma+1$, the reversed inequality holds. 
Here the inequalities of (ii) are sharp if $\alpha, \beta, \gamma$ are allowed to range as specified. For restricted ranges of $\alpha, \beta, \gamma$, the results of (iii)-(v) provide better estimates.

4. It is familiar that many standard integrals can be obtained by transformation of the integral (2). In particular, by substituting $t=\cos ^{2} \frac{1}{2} \theta$ and $t=4 \rho /(1+\rho)^{2}$ in (2) we obtain the formulae

$$
\begin{aligned}
\int_{0}^{\pi}\left(1-2 \rho \cos \theta+\rho^{2}\right)^{-\alpha}\left(\sin \frac{1}{2} \theta\right)^{2 \beta-1}\left(\cos \frac{1}{2} \theta\right)^{2 \gamma-1} d \theta & \\
= & (1+\rho)^{-2 \alpha} I\left(\alpha, \beta, \gamma, 4 \rho /(1+\rho)^{2}\right) \quad(0 \leqq \rho<1),
\end{aligned}
$$

and

$$
\begin{aligned}
\int_{0}^{1}\left(1-2 \rho \cos \theta+\rho^{2}\right)^{-\alpha}(1-\rho)^{2 \beta-1} \rho^{\gamma-1}(1+\rho)^{2 \alpha-2 \beta-2 \gamma+1} d \rho & \\
& =4^{-\gamma} I\left(\alpha, \beta, \gamma, \cos ^{2} \frac{1}{2} \theta\right) \quad(\theta \text { real }) .
\end{aligned}
$$

In combination with our theorem, these give rise to several useful inequalities. Again, by substituting $t=1-v / s, x=\delta /(\delta+v)$, where $v>0, \delta>0$, we see that

$$
\int_{0}^{\infty}(\delta+s)^{-\alpha} s^{\alpha-\beta-1} d s=v^{\alpha-\beta}(v+\delta)^{-\alpha} I(\alpha, \beta, 1, \delta /(\delta+v)) .
$$

Taking $\delta=1-\rho$, we may deduce (cf. 4, Lemma 1) that, if $0 \leqq \rho<1$ and $0 \leqq v \leqq \pi$, then

$$
\int_{0}^{\pi}\left|1-\rho e^{i \theta}\right|^{-\alpha} \theta^{\alpha-\beta-1} d \theta \leqq A(\alpha, \beta)\left|1-\rho e^{i v}\right|^{-\beta},
$$

where $A(\alpha, \beta)$ is a positive constant depending only on $\alpha, \beta$.

Finally, by putting $\rho=\left(e^{\zeta}-1\right) /\left(e^{\zeta}+1\right)$, where $\zeta>0$, in the first formula of this section, and using Laplace's second integral for the Legendre polynomial (1, p. 277), we have

$$
P_{n}(\cosh \zeta)=\pi^{-1} e^{-(n+1) \zeta} I\left(n+1, \frac{1}{2}, \frac{1}{2}, 1-e^{-2 \zeta}\right) .
$$

The theorem thus gives, for $\zeta>0$,

$$
P_{n}(\cosh \zeta) \leqq e^{n \zeta}\left\{e^{-2 \zeta}+\left(1-e^{-2 \zeta}\right) \frac{(2 n) !}{2^{2 n}(n) !^{2}}\right\} \leqq e^{n \zeta},
$$

an improvement on a result stated in (4).

5. I remark in conclusion that the referee has pointed out to me that a selfcontained proof of the theorem in $\S 3$ may be obtained via the formula

$$
I(\alpha, \beta, \gamma, x)=(1-x)^{\beta-\alpha} \int_{0}^{1}(1-u x)^{\alpha-\beta-\gamma}(1-u)^{\gamma-1} u^{\beta-1} d u,
$$

E.M.S. - C 
where $0 \leqq x<1$ (this follows from (2) by the substitution $t=(1-u) /(1-u x)$ ). Here the coefficient of $(1-x)^{\beta-\alpha}$ is a convex function of $x$ if $\alpha<\beta+\gamma$ or $\alpha>\beta+\gamma+1$.

I am also indebted to the referee for other helpful remarks.

\section{REFERENCES}

(1) E. T. Copson, An Introduction to the Theory of Functions of a Complex Variable (Oxford, 1935).

(2) T. ERBER, Inequalities for hypergeometric functions, Arch. Rational Mech. and Anal. 4 (1959-60), 341-351.

(3) A. ERDÉlyi (Ed.), Higher Transcendental Functions, Vol. I, Bateman Manuscript Project (McGraw-Hill, 1953).

(4) T. M. FLetr, Some theorems on fractional integrals, Proc. Cambridge Philos. Soc. 55 (1959), 31-50.

(5) T. M. FLetr, A theorem on functions of class $H^{\lambda}$, Proc. London Math. Soc. (3) 14A (1965), 86-92.

(6) T. M. FLETT, Mean values of power series, Pacific J. Math. 25 (1968), 463-494.

Department of Pure Mathematics

THE UNIVERSITY

SHEFFIELD 